\title{
Sample preparation method to improve the efficiency of high-throughput single-molecule force spectroscopy
}

\author{
Lei Jin ${ }^{1,3}$, Li Kou ${ }^{1,3}$, Yanan Zeng ${ }^{2}$, Chunguang $\mathrm{Hu}^{1,3}$, Xiaodong $\mathrm{Hu}^{1,3}$ \\ 1 State Key Laboratory of Precision Measuring Technology \& Instruments, Tianjin University, Tianjin 300072, China \\ 2 College of Engineering and Technology, Tianjin Agricultural University, Tianjin 300384, China \\ 3 Nanchang Institute for Microtechnology of Tianjin University, Tianjin 300072, China
}

Received: 28 November 2018 / Accepted: 28 January 2019 / Published online: 1 October 2019

\begin{abstract}
Inefficient sample preparation methods hinder the performance of high-throughput single-molecule force spectroscopy (H-SMFS) for viscous damping among reactants and unstable linkage. Here, we demonstrated a sample preparation method for H-SMFS systems to achieve a higher ratio of effective target molecules per sample cell by gas-phase silanization and reactant hydrophobization. Digital holographic centrifugal force microscopy (DH-CFM) was used to verify its performance. The experimental result indicated that the DNA stretching success ratio was improved from $0.89 \%$ to $13.5 \%$. This enhanced efficiency preparation method has potential application for force-based DNA stretching experiments and other modifying procedures.
\end{abstract}

Keywords Specific binding, Digital holographic centrifugal force microscopy, Sample preparation, High-throughput single-molecule force spectroscopy

\section{INTRODUCTION}

High-throughput single-molecule force spectroscopy (HSMFS) systems are developed to shorten the SMFS experiment period and enhance resolution within limited time via statistical algorithms (Neuman and Nagy 2008). All H-SMFS systems, such as magnetic tweezers and acoustic tweezers, have the potential to manipulate hundreds of molecules simultaneously (Johnson et al. 2017; Lu et al. 2017; Madariaga-Marcos et al. 2018). Normal SMFS systems, such as optic tweezers (OT) and atomic force microscopy (AFM), have the ability to control specific individual molecules with higher strength often more than $100 \mathrm{pN}$ and even $300 \mathrm{pN}$ (Bennink et al. 1999). Thus, molecules can be preprocessed if attached to the substrate. However, for H-SMFS systems, especially for CFM, the maximal force is often lower than $60 \mathrm{pN}$ (Halvorsen and Wong 2010b) and it is difficult to adjust the spatiality of tethered beads. So,

$\bowtie$ Correspondence: xdhu@tju.edu.cn (X. Hu) experiments with H-SMFS systems often rely on sample quality, which indicates the ratio of beads that can be stretched freely. However, there exist only a few specific studies on sample preparation methods for H-SMFS. According to former experiments, the ratios of wellstretched molecules in a sample cell are often lower than 3\% (Hill et al. 2017), which directly impair the throughput of H-SMFS systems.

The poor efficiency of these samples could mainly be attributed to the following reasons: hydrophilicity and non-specificity. Functioned beads are commonly used as the measurement medium in SMFS. In order to tether them on one end of the molecule with biotin, these beads are routinely coated with streptavidin. Digoxigenin and its antibody are also used in protocols for the same reason (Yokokawa et al. 2008). All these reactants are hydrophilic. The staple component of coverslips and beads in this experiment is silicon dioxide. Due to the hydrophilicity, streptavidin-coated beads tend to be attracted and even adhere to the anti-digoxigenincoated coverslips. Due to this, a large number of 
molecules cannot be stretched freely in the processes of H-SMFS experiments. Additionally, the concentration of anti-digoxigenin contributes to this adhesion problem which will be further discussed later. To tether molecules, anti-digoxigenin is often injected directly in the sample cell and is bonded on the substrate nonspecifically due to which, the bonding strength is difficult to evaluate (Yang et al. 2016a).

To solve these problems, Halvorsen et al. (2011) proposed the use of DNA self-assembly techniques to form a force-activated single-molecule switch. However, because of the complexity of nanoengineering, it is difficult to implement in a normal experimental environment. Silane coupling agents have the ability to form a durable bond between organic and inorganic materials (Di et al. 2016; Kango et al. 2013), which are widely used in surface treatment to obtain specific functions such as dielectric properties (Pan et al. 2017), superwetting surfaces (Shi et al. 2016), and natural fiber/ polymer composites (Xie et al. 2010). It is a mature technique as a surface conditioning method (Lung and Matinlinna 2012), and its performance can be enhanced by gas-phase silanization (Fan and Lopinski 2010; Steinbach et al. 2016). Bovine serum albumin (BSA) is always added into the buffer to separate beads, but its hydrophobicity is not sufficient to resist the adhesion problem between beads and the substrate. Notably, not all silane coupling agents fit the H-SMFS. Hydrophilic reactants such as 7-Octen-1-yl $\left(\mathrm{CH}_{2}=\mathrm{CH}\right.$-terminal $)$ and 3-(2-aminoethyl) aminopropyl $\quad\left(\mathrm{NH}_{2}\right.$-terminal) trimethoxysilanes exacerbate the adhesion problem (Kwak et al. 2003a, b; 2004).

Here, we demonstrate a sample preparation method for H-SMFS systems that improves experimental efficiency by modifying the substrate and utilizing the specified linker and hydrophobic silane coupling agents. To form a reliable monomer silane film on coverslips, gas-phase silanization technique is used. The experiment success ratio is verified by digital holographic centrifugal force microscopy (DH-CFM), an improved version of CFM proposed by Halvorsen and Wong (2010a). After performing 200 experiments, the results indicated that the experimental success ratio obtained by the proposed method was approximately ten times higher than that obtained by the method widely used in H-SMFS, such as magnetic tweezers (Kreft et al. 2018; Kriegel et al. 2017, 2018; Lipfert et al. 2010) and CFM (Hoang et al. 2016; Yang et al. 2016b).

\section{RESULTS}

Several experiments were carried out to verify the performance of this sample preparation method. In this part, the effect of silanization was verified compared to the normal method. We analyzed the result of variable controlled samples with different concentrations of antidigoxigenin. Moreover, DH-CFM was introduced to examine the comprehensive response of the sample following this new enhanced efficiency method.

\section{Reaction effect}

Conglutination and specificity are major problems of the sample preparation methods, which cause low experimental throughput. To solve these problems, we functionalized coverslips with hydrophobic silane (MPTES and MTES). The results of the experiment are shown in Fig. 1.

Beads are injected into two different sample cells (one is silanized and the other is not), respectively, without DNA molecules. As shown in Fig. $1 \mathrm{~A}$ and B, fewer beads adhered on the substrate after silanization compared to those with the normal substrate. This means that the monomolecular layer of silane prevents the beads from being stuck to the coverslip surface. However, for the adhesion problem, there are still some reactants, in following steps, which could influence the
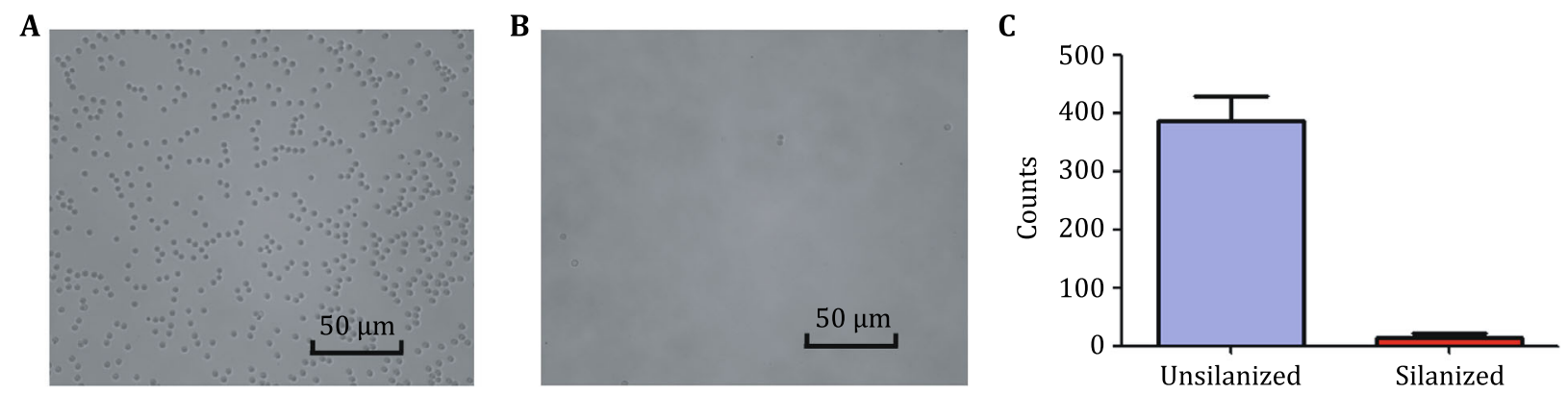

Fig. 1 Performance of gas-phase silanization. A Raw sample image without silanization. B Raw sample image after silanization. The condition as well as reaction process of both $\mathbf{A}$ and $\mathbf{B}$ are totally the same except for silanization. $\mathbf{C}$ Statistical comparison of the result of silanization 
property of the coverslips' surface. Anti-digoxigenin, as a typical substance, tends to attract beads and the adhesion ability depends on its concentration. Figure 2 indicates that the proportion of adhered beads increases with the concentration of anti-digoxigenin. Therefore, we assume that there is a positive correlation between the adhesion ratio and the concentration of antidigoxigenin. Anti-digoxigenin reacts with SMCC and DNA. Thus, its concentration is relevant to the distribution of the linked beads. To reduce the adhesion proportion and cover all the SMCC bonded to the substrate, a concentration of $3 \%$ was selected.

\section{Mechanical analysis}

To verify the effectiveness of this new method, digital holographic centrifuge force microscopy (DH-CFM) was introduced.

Mechanical property verification requires the sample cell to be placed vertically in the DH-CFM. Every bead will be influenced by the centrifugal force field as shown in Fig. 3. The resultant force of the tethered bead can be described as
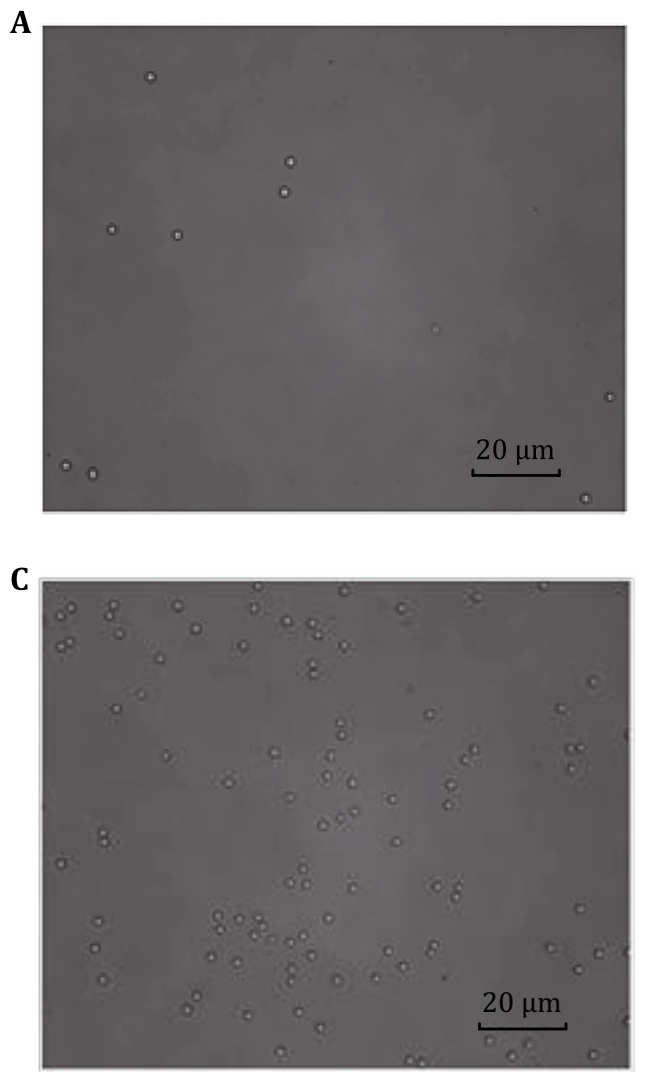

$F=-T_{m}=\frac{f_{c}-f_{v}}{\sin \varphi}+\frac{g-f_{\beta}}{\cos \varphi}$

where $\varphi$ is the angle between the coverslip and the nucleic acid, and $f_{c}, f_{v}, f_{\beta}$, and $g$ represent the centrifugal force, viscous force, buoyancy, and gravity, respectively. With dynamic centrifugal forces, the gravity and buoyancy are immutable. The beads involved in this experiment are made of silica coated by streptavidin (the density of silica is $2.2 \mathrm{~g} / \mathrm{cm}^{3}$ at room temperature) with a mean diameter of $4.86 \mu \mathrm{m}$. The density of PBS in the sample cell is $1.005 \mathrm{~g} / \mathrm{cm}^{3}$. Thus, the gravity and buoyancy are $\sim 2.5254 \times 10^{-4} \quad \mathrm{pN}$ and $\sim 1.1536 \times 10^{-4} \mathrm{pN}$, respectively. Due to the stretching mode of DH-CFM, viscous force would be within $\sim 1 \times 10^{-4} \mathrm{pN}$. However, the centrifugal force ranges from 0 to $71.5 \mathrm{pN}$. Hence, the viscous force, gravity, and buoyancy are small enough to be neglected.

\section{Efficiency analysis}

During the experiment, well-linked beads can be stretched away from the substrate and then kept at a specific

B

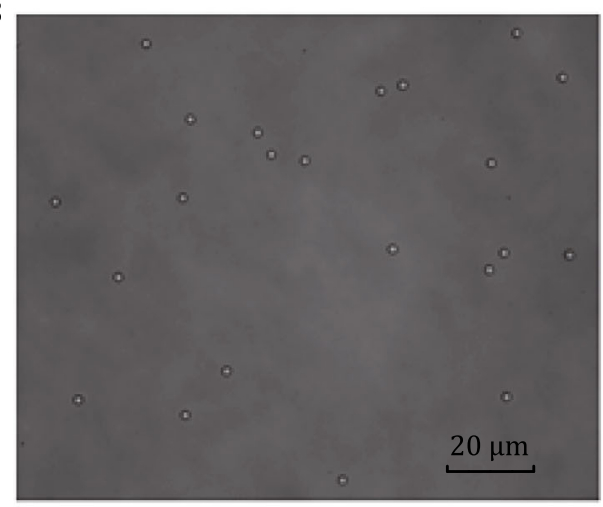

D

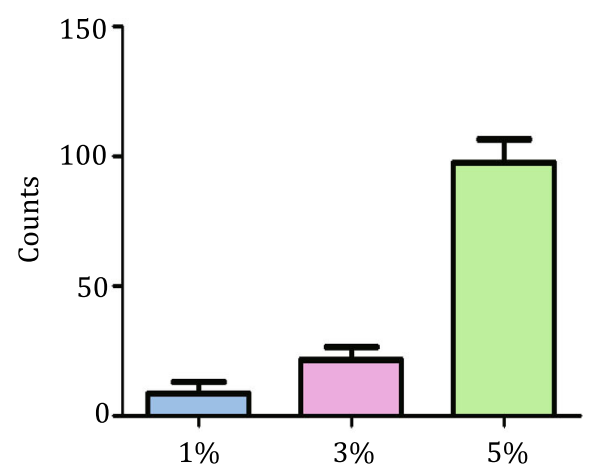

Fig. 2 Experimental data. A, B, C Typical raw images of sample cells in which the concentrations of anti-digoxigenin are 1\%, 3\%, and 5\%, respectively. $\mathbf{D}$ Counts of the beads adhered in sample cells with different concentrations of anti-digoxigenin 


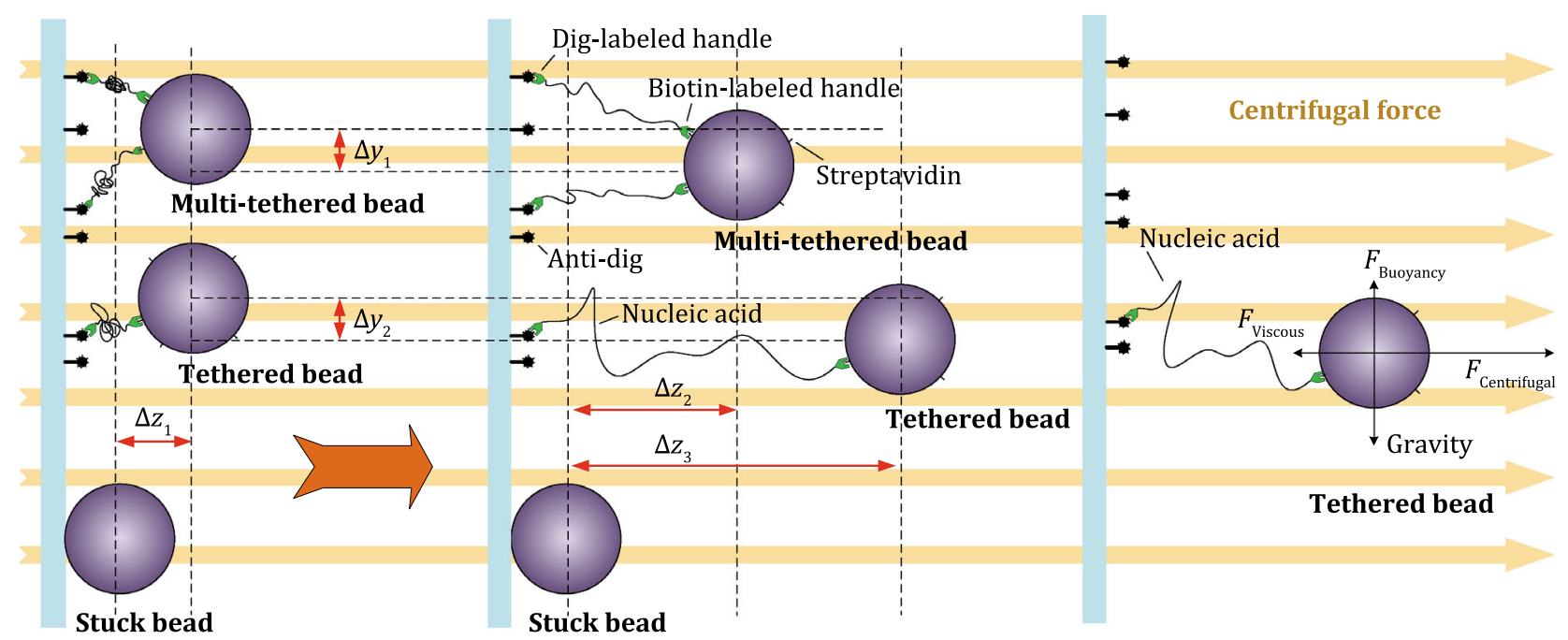

Fig. 3 State space of particles. Because of the scale difference between nucleic acids and the link joint, the beads are linked with two or more molecules simultaneously. Four different kinds force will work on the tethered beads, but they are small enough to be neglected

distance. They can be discerned easily among stuck beads through the intensity distribution of the beam.

As shown in Fig. 4A, beads can be separated into three groups according to their state: (a) well stretched, (b) sub-stretched, and (c) stuck on the substrate. Substretched beads are tethered by two or more molecules simultaneously and so, their extension level is lower, as seen in group b. Furthermore, for the reasons mentioned in the method part, some beads still tend to be stuck on the substrate. However, this problem has been obviously improved as shown in Fig. 4B. The ratios of freely stretched beads in the above experiments are $15.7 \%$ and $1.7 \%$ (including sub-stretched beads), respectively.

The common method here is cited from the methods often used in magnetic tweezers (Kreft et al. 2018; Kriegel et al. 2017, 2018; Lipfert et al. 2010) and is also mentioned in the CFM system (Hoang et al. 2016; Yang et al. 2016b). The result following this common method has been shown in Fig. 4B. In this contrast group, antidigoxigenin is directly injected into the sample cell cleaned using an ultrasonic cleaner in ultrapure water and in $75 \%$ ethanol, respectively. Then, the DNA molecules and streptavidin-coated beads are added. PBS with BSA and Tween-20 are used as the solvents in the sample cell. The process of reactant addition is the same as the proposed method mentioned before. To obtain more experimental data, twenty samples can be prepared simultaneously as an experimental group and ten groups are implemented using each method. Thus, two hundred H-SMFS experiments for each type of protocol were implemented. Using the same algorithm as in Fig. $4 \mathrm{~A}$ and B, every experiment success ratio has been determined, as shown in Fig. 4C. The average experiment success ratio with the two types of methods is $13.5 \%$ (by proposed method) and $0.89 \%$ (by common method), which indicates that the efficiency has been improved by more than ten times.

\section{Stretching experiment}

Using DNA molecules with a length of $11.92 \mathrm{kbp}$ $(\sim 3.58 \mu \mathrm{m})$ as the stretched material, DH-CFM can obtain an extension curve with dynamic centrifugal forces, as shown in Fig. 4D.

Force-extension curves of ten beads have been obtained. The length of the DNA molecules tends to be extended from $\sim 3.7$ to $\sim 5 \mu \mathrm{m}$ in the centrifuge force field. Because of the limitation of the CFM's stretching ability (the highest rotary speed is $3500 \%$ s), the highest centrifugal force is $71.5 \mathrm{pN}$. As per the results of the stretching experiment shown in Fig. 4D, the average extension curve accords with the data provided by Smith et al. (1996) and fits the worm-like chain with the persistent length of $50 \mathrm{~nm}$ (Abels et al. 2005; Kirby 2010).

The DNA extension curves are obtained via the method of radius vector projection which detects changes in the diffraction rings of the microparticles at different distances from substrate. As shown in Fig. 4A, the rings of particles, which have been stretched successfully, are different compared to others. However, DNA overstretching transition does not occur at $\sim 65 \mathrm{pN}$ and just shows a similar tendency over $70 \mathrm{pN}$. This may due to the measurement deficiency in the present version of the DH-CFM system. Thus, additional 
A

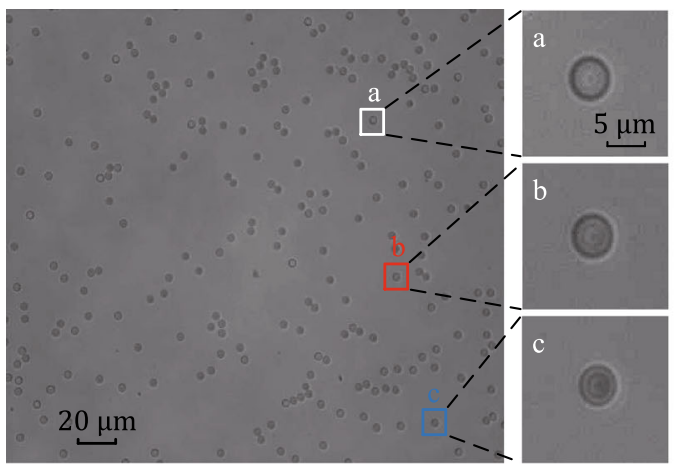

C

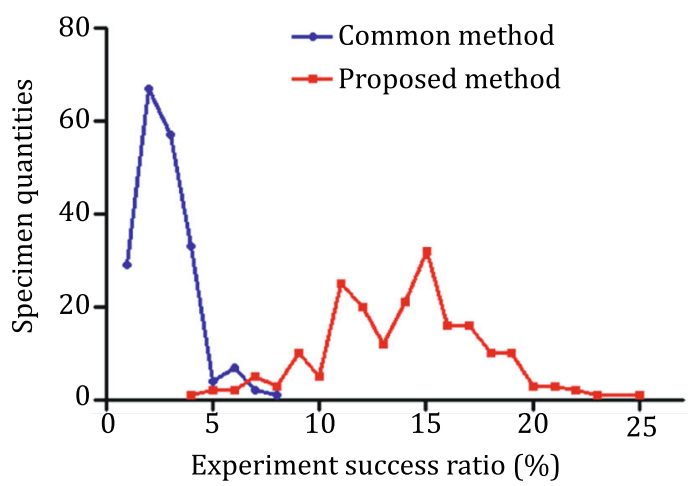

B

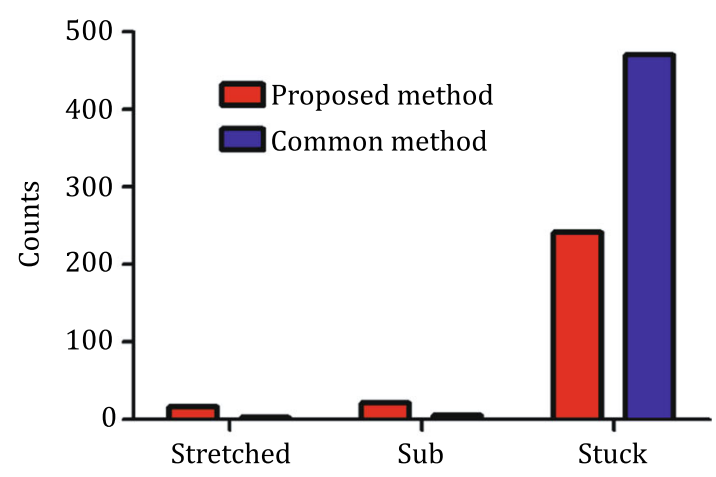

D

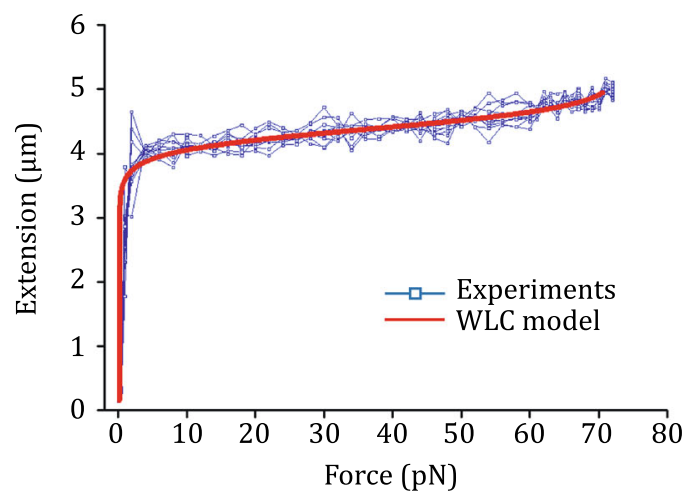

Fig. 4 Performance of the proposed method. A Raw sample image after treatment by the proposed method. B Comparison of the specific performance between the proposed and common method; the common method here has been described in the manuscript. C Experimental success ratio distribution in these two different methods. D Force-extension curves of $11.92 \mathrm{kbp}$ DNA molecules tethered to $4.86 \mu \mathrm{m}$ silica beads

discussion is needed, but it is enough to verify the performance of the proposed sample preparation method.

\section{DISCUSSION}

The proposed method provides a specific ligation and a hydrophobic substrate that maintains the throughput of the H-SMFS system. In this manner, the experiment success ratio is improved from $0.85 \%$ to $13.5 \%$. This method also enhances the predictability including the resolution of SMFS systems, and the strength of every chemical bond involved in the sample cell can be evaluated because of the specific linkage. As shown in Fig. 5, the layered structure permits the reactant to perform separately, which means that reactants on the upperlayer would not influence the sub-layer. There is a step of removing extra reactants between layers. It is worth noting that we have compared the performance of gasphase silanization with liquid-phase silanization such as sigmacote. Gas-phase silanization is much more stable and reliable than liquid-phase silanization because the silane coupling agent distributes more evenly in vacuum. Due to the classic way of DNA linking and the normal silane coupling agent, all reactants involved in this method are available commercially. Thus, the experiment condition can be provided by most SMFS laboratories. Moreover, the function of the proposed method would not be influenced by the shape or size of the sample cell, and could be applied in various SMFS systems.

The work mentioned above is part of a DH-CFM project, which is a developing system. Thus, the DNA extension curves are inconsistent with the previous study that there should be an overstretching transition at $\sim 65 \mathrm{pN}$. This may be because of the measurement lag. Fortunately, the result of the stretching experiment is sufficient to verify the performance of the proposed method. During the sample preparation method implementing process, each reactant is ideally supposed to be hydrophobic. However, some functional reactants such as streptavidin and digoxigenin are not supposed to be substituted. So, the adhesion phenomenon is difficult to 


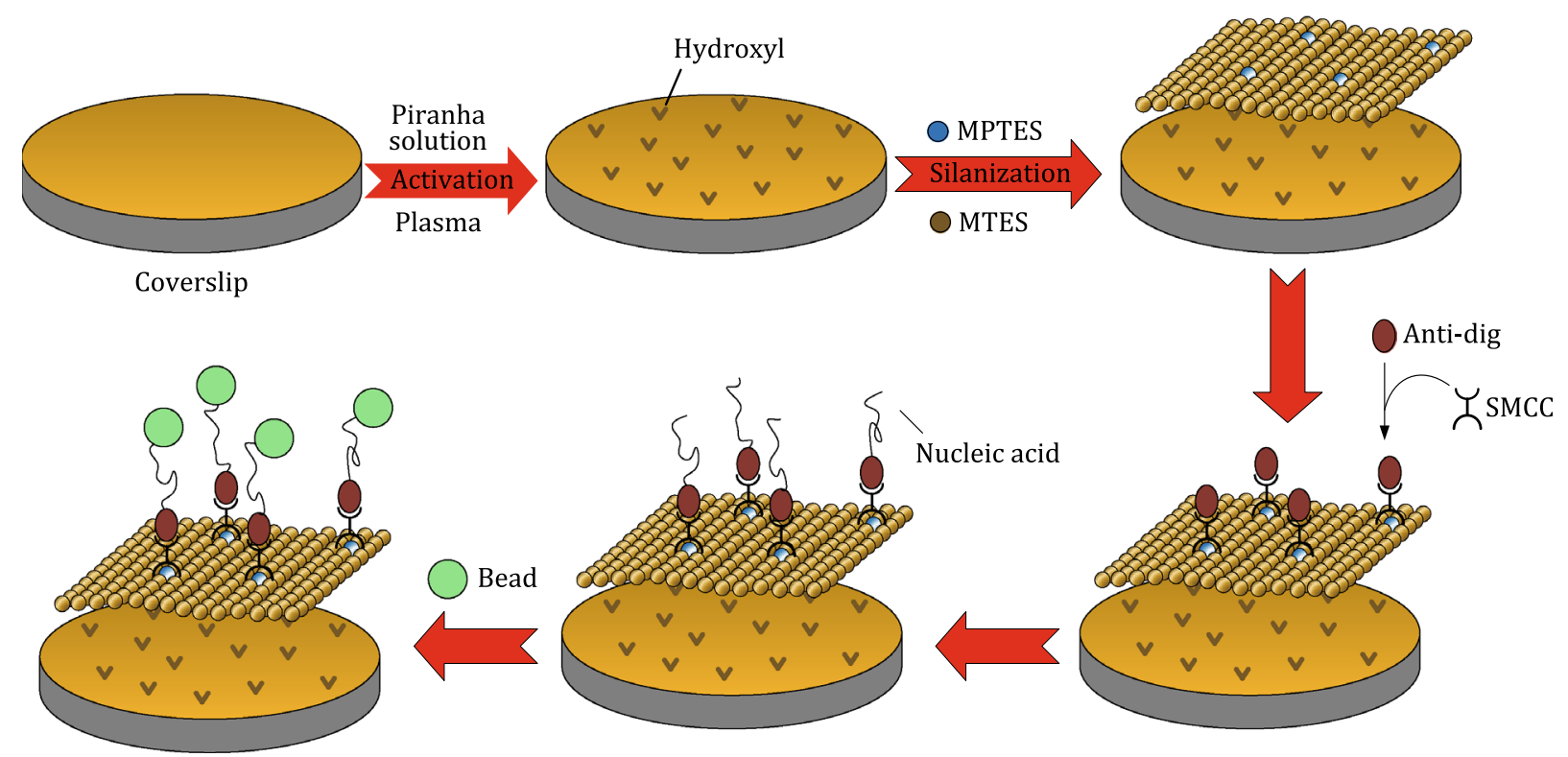

Fig. 5 Schematic diagram of complete linkage. The complete process of this treatment may last 2 days. The sample cell needs to be rinsed with PBS or another solution after each step to remove the extra reactant

be eliminated. We found that streptavidin-coated beads had the same problem of adhesion in other experiments as well. Although bovine serum albumin and Tween-20 are already added to the solvent of beads when they are produced, it is not enough to separate the beads perfectly because of the hydrophilic nature of streptavidin. Apart from the reactants, there may be other reasons for the adhesion problem, such as electrostatic force.

\section{MATERIALS AND METHODS}

The structure of a simple cell for the DH-CFM in this study was composed of two coverslips with diameters of 10 and $24 \mathrm{~mm}$ (Marienfeld) as well as two pieces of parafilm (Bemis). In this structure, the channel formed between the two pieces of parafilm provided a reaction zone for subsequent linkages. The complete structure of the linkage is shown in Fig. 5. Epoxy resin was used to seal the two ports of the channel after the linking process. The volume of this sample cell was $\sim 10 \mu \mathrm{L}$ and its height $\sim 200 \mu \mathrm{m}$. The proposed method does not rely on any specific structure and so, the sample cell is changeable for different equipment.

The implementation can be divided into two main steps: (1) surface modification and (2) molecular ligation.

The main purpose of surface modification is to enhance the hydrophobicity of coverslips to reduce the ratio of adhered beads, as shown in Fig. 1 . Silane is a reagent commonly used for surface modification because of its simple preparation process and reliable performance. Before the modifying process, coverslips are supposed to be cleaned with an ultrasonic-cleaner in ultrapure water and with $75 \%$ ethanol, respectively. Each cleaning step is supposed to last for more than $30 \mathrm{~min}$ to remove dust and organic impurities. To hydroxylate the substrate, deeper cleaning and activation of coverslips is carried out via dipping in a piranha solution (mixture of $70 \%$ sulfuric acid and $30 \%$ hydrogen peroxide) for $15 \mathrm{~min}$ and exposing to plasma cleaner for $5 \mathrm{~min}$. In this manner, a new functional group-hydroxyl-is generated on the surface of the coverslips. Liquid silane can be easily vaporized in vacuum, diffusing evenly to the entire space. This process should best be sustained for more than $8 \mathrm{~h}$. Then, these coverslips should be washed thoroughly with acetone to remove the extra silane and then rinsed with ethanol to eliminate the extra acetone. Excessive ethanol will be vaporized spontaneously. In some cases, before the washing process, the monolayer can be solidified by baking for $15 \mathrm{~min}$ at $90^{\circ} \mathrm{C}$.

A high-efficiency sample preparation method needs to guarantee that every reactant is linked specifically so that all the mechanical and physical properties of each bonding joint can be evaluated. Anti-digoxigenin is the most common intermediate reactant between the substrate and DNA. It is also involved in this experiment but in a different way. Anti-digoxigenin, as a kind of protein, has an important group. amidogen. This enables it to bond with 4-( $\mathrm{N}$-maleimidomethyl)-cyclohexane-1carboxylate (SMCC, HEOWNS). As SMCC is insoluble in 
water, dimethyl sulfoxide (DMSO) is introduced in this method. SMCC needs to be first dissolved in DMSO at a concentration of $3.6 \mathrm{mg} / \mathrm{mL}$, and then mixed with antidigoxigenin at room temperature for $30 \mathrm{~min}$ or at $4{ }^{\circ} \mathrm{C}$ for $60 \mathrm{~min}$. Subsequently, it needs to be purified to eliminate excessive SMCC. Purified protein should be attenuated with phosphate-buffered saline (PBS) (137 mmol/L NaCl, $10 \mathrm{mmol} / \mathrm{L}$ phosphate, $2.7 \mathrm{mmol} / \mathrm{L}$ $\mathrm{KCl}, \mathrm{pH}$ 7.4) and injected in sample cells. The specific concentration will be discussed in the experimental part. Purified protein will react with 3-mercaptopropyltriethoxysilane (MPTES, HEOWNS), which has been bonded on the coverslip surface of coverslips. This reaction should be sustained $30 \mathrm{~min}$ at $20{ }^{\circ} \mathrm{C}$. The DNA $(10 \mu \mathrm{L}$ of $0.2 \mathrm{ng} / \mu \mathrm{L})$ and functionalized beads $(10 \mu \mathrm{L}$ in density of $0.5 \mathrm{~g} / \mathrm{cm}^{3}$ ) are routinely injected into the sample cell, respectively. The beads involved in this experiment are streptavidin-coated silica microparticles (Bangs Laboratories Inc.) with a diameter of $5 \mu \mathrm{m}$. Both the injecting steps mentioned above are supposed to sustain $30 \mathrm{~min}$ at room temperature. Before injecting the beads, superfluous DNA molecules would better be eliminated by rinsing with PBS. Thus, PBS is the only solvent in the sample channel, which will be helpful for the subsequent optical detection.

It is not unusual that one bead is linked by two or more molecules. Thus, the data of the stretching process may not be precise enough. To solve this problem, the distance among molecules has to be adjusted. With the benefits of specific binding, the interspace among antidigoxigenin molecules can be modulated by adjusting the spatial distribution of silane, ignoring the characteristics of the molecule itself. Therefore, triethoxymethylsilane (MTES, TCI) is introduced. MTES is a stable reactant that does not participate in the reaction and is merely used to supplement the extra space among MPTES. In this manner, the distribution of antidigoxigenin can be adjusted by changing the proportion of these two silanes. The percentage of ten is not unacceptable (for example: $5 \mu \mathrm{L}$ MPTES in $45 \mu \mathrm{L}$ MTES).

However, there is the problem that anti-digoxigenin tends to attract functional beads in liquid condition so that the linkage system is easily stuck on the substrate rather than being stretched freely in the centrifugal force field during experiment, leading to low throughput. As shown in Fig. 2, there is a positive correlation between the adhesion ratio and the concentration of anti-digoxigenin. This means that the concentration of anti-digoxigenin will directly influence the result and should be well evaluated. Optimization of the antidigoxigenin concentration is discussed in the experimental part.
Acknowledgements This work was financially supported by the National Nature Science Foundation of China (51775381), National Major Scientific Instruments and Equipment Development Special (2017YFF0107001), and The Tianjin Research Program of Application Foundation and Advanced Technology (15JCZDJC3160).

\section{Compliance with Ethical Standards}

Conflict of interest Lei Jin, Li Kou, Yanan Zeng, Chunguang $\mathrm{Hu}$, and Xiaodong $\mathrm{Hu}$ declare that they have no conflicts of interest.

Human and animal rights and informed consent This article does not contain any studies with human or animal subjects performed by any of the authors.

Open Access This article is distributed under the terms of the Creative Commons Attribution 4.0 International License (http:// creativecommons.org/licenses/by/4.0/), which permits unrestricted use, distribution, and reproduction in any medium, provided you give appropriate credit to the original author(s) and the source, provide a link to the Creative Commons license, and indicate if changes were made.

\section{References}

Abels JA, Moreno-Herrero F, Van der Heijden T, Dekker C, Dekker $\mathrm{NH}$ (2005) Single-molecule measurements of the persistence length of double-stranded RNA. Biophys J 88:2737-2744

Bennink ML, Schärer OD, Kanaar R, Sakata-Sogawa K, Schins JM, Kanger JS, de Grooth BG, Greve J (1999) Single-molecule manipulation of double-stranded DNA using optical tweezers: interaction studies of DNA with RecA and YOYO-1. Cytometry 36:200-208

Di X, Zhang W, Zang D, Liu F, Wang Y, Wang C (2016) A novel method for the fabrication of superhydrophobic nylon net. Chem Eng J 306:53-59

Fan C, Lopinski G (2010) STM and HREELS investigation of gas phase silanization on hydroxylated Si (100). Surf Sci 604:996-1001

Halvorsen K, Wong WP (2010) Massively parallel single-molecule manipulation using centrifugal force. Biophys J 98:L53-L55

Halvorsen K, Schaak D, Wong WP (2011) Nanoengineering a single-molecule mechanical switch using DNA self-assembly. Nanotechnology 22:494005

Hill FR, Monachino E, van Oijen AM (2017) The more the merrier: high-throughput single-molecule techniques. Biochem Soc Trans 45:759-769

Hoang T, Patel DS, Halvorsen K (2016) A wireless centrifuge force microscope (CFM) enables multiplexed single-molecule experiments in a commercial centrifuge. Rev Sci Instrum 87:083705

Johnson KC, Clemmens E, Mahmoud H, Kirkpatrick R, Vizcarra JC, Thomas WE (2017) A multiplexed magnetic tweezer with precision particle tracking and bi-directional force control. J Biol Eng 11:47

Kango S, Kalia S, Celli A, Njuguna J, Habibi Y, Kumar R (2013) Surface modification of inorganic nanoparticles for development of organic-inorganic nanocomposites-a review. Prog Polym Sci 38:1232-1261

Kirby BJ (2010) Micro- and nanoscale fluid mechanics: transport in microfluidic devices. Cambridge University Press, Cambridge 
Kreft D, Wang Y, Rattay M, Toensing K, Anselmetti D (2018) Binding mechanism of anti-cancer chemotherapeutic drug mitoxantrone to DNA characterized by magnetic tweezers. J Nanobiotechnol 16:56

Kriegel F, Ermann N, Forbes R, Dulin D, Dekker NH, Lipfert J (2017) Probing the salt dependence of the torsional stiffness of DNA by multiplexed magnetic torque tweezers. Nucleic Acids Res 45:5920-5929

Kriegel F, Vanderlinden W, Nicolaus T, Kardinal A, Lipfert J (2018) Measuring single-molecule twist and torque in multiplexed magnetic tweezers. Methods Mol Biol 1814:75-98

Kwak K, Kudo H, Fujihira M (2003a) Imaging stretched single DNA molecules by pulsed-force-mode atomic force microscopy. Ultramicroscopy 97:249-255

Kwak K, Yoda S, Fujihira M (2003b) Observation of stretched single DNA molecules by Kelvin probe force microscopy. Appl Surf Sci 210:73-78

Kwak K, Sato F, Kudo H, Yoda S, Fujihira M (2004) Topographic effects on adhesive force mapping of stretched DNA molecules by pulsed-force-mode atomic force microscopy. Ultramicroscopy 100:179-186

Lipfert J, Kerssemakers JWJ, Jager T, Dekker NH (2010) Magnetic torque tweezers: measuring torsional stiffness in DNA and RecA-DNA filaments. Nat Methods 7:977-980

Lu X, Soto F, Li J, Li T, Liang Y, Wang J (2017) Topographical manipulation of microparticles and cells with acoustic microstreaming. ACS Appl Mater Interfaces 9:38870-38876

Lung CYK, Matinlinna JP (2012) Aspects of silane coupling agents and surface conditioning in dentistry: an overview. Dent Mater 28:467-477
Madariaga-Marcos J, Hormeño S, Pastrana C, Fisher G, Dillingham M, Moreno-Herrero F (2018) Force determination in lateral magnetic tweezers combined with TIRF microscopy. Nanoscale 10:4579-4590

Neuman KC, Nagy A (2008) Single-molecule force spectroscopy: optical tweezers, magnetic tweezers and atomic force microscopy. Nat Methods 5:491

Pan C, Kou K, Jia Q, Zhang Y, Wu G, Ji T (2017) Improved thermal conductivity and dielectric properties of hBN/PTFE composites via surface treatment by silane coupling agent. Compos B Eng 111:83-90

Shi H, He Y, Pan Y, Di H, Zeng G, Zhang L, Zhang C (2016) A modified mussel-inspired method to fabricate $\mathrm{TiO}_{2}$ decorated superhydrophilic PVDF membrane for oil/water separation. J Membr Sci 506:60-70

Smith SB, Cui Y, Bustamante C (1996) Overstretching B-DNA: the elastic response of individual double-stranded and singlestranded DNA molecules. Science 271:795-799

Steinbach AM, Sandner T, Mizaikoff B, Strehle S (2016) Gas phase silanization for silicon nanowire sensors and other lab-on-achip systems. Physica Status Solidi (c) 13:135-141

Xie Y, Hill CA, Xiao Z, Militz H, Mai C (2010) Silane coupling agents used for natural fiber/polymer composites: a review. Compos Part A 41:806-819

Yang D, Ward A, Halvorsen K, Wong WP (2016) Multiplexed singlemolecule force spectroscopy using a centrifuge. Nat Commun 7:11026

Yokokawa R, Miwa J, Tarhan MC, Fujita H, Kasahara M (2008) DNA molecule manipulation by motor proteins for analysis at the single-molecule level. Anal Bioanal Chem 391:2735 